\title{
Standing Together: How Bioethics and Public Health Can Join Forces to Provide Equitable Health Care
}

\author{
Jennifer Bard, J.D., M.P.H.,Texas Tech University School of Law
}

The tragic displacement of the poorest citizens of New Orleans in the wake of Hurricane Katrina is a tragedy, but also an opportunity. The public is seeing the terrible impact of economic equality in the United States, and are expressing their shame and dismay. One of the most pressing needs following the hurricane has been the provision of adequate health care. From the moment that people started seeking shelter in the New Orleans Superdome two things became clear. First, the stadium lacked the sanitary resources to prevent the spread of infectious disease. Second, the population that sought refuge in the Superdome represented some of the poorest in New Orleans, people whose inability to pay for medical care resulted in their being severely impaired by a range of untreated chronic diseases such as high blood pressure and diabetes. Neither of these realizations should come as any surprise to people familiar with the shameful allocation of health care resources in this country. The patchwork of government and private health insurance still leaves 44 million Americans without access to the kinds of routine health care that can prevent, diagnose, manage or effectively treat illness.

From the perspective of a person with a foot in both the bioethics and the public health sphere, I believe that Jonathan Moreno's (2005) call for bioethics to become more involved in the broader environmental movement also highlights the need for bioethics and public health to join forces and address the lack of universal access to quality health care. Bioethicists and public health experts already recognize the reality of limited health care resources and the problems that can result, but there is substantially more we can do to address the underlying inequalities that lead to public health disasters like the one in New Orleans. For example, as a bioethicists and public health practitioners we may wrestle with the question of whether a prisoner with advanced Hepatitis C-related disease is a good candidate for a liver transplant. If the prisoner had had access to early testing and treatment, however, the transplant in all likelihood would not be necessary.

Early analysis of the events following Hurricane Katrina and the resulting levee failure clearly show that it was the poor who bore the brunt of the failure to adequately maintain the flood prevention infrastructure. Not only were the poorest neighborhoods of New Orleans located in lowlying areas that are the particularly susceptible to flooding, but the residents of these neighborhoods were also the most likely to lack adequate insurance. Public health practitioners and scholars have long known that it is the poor who bear the brunt of dangerous environmental conditions. They are the most likely to live in the shadow of factories and power plants, or in houses built on contaminated soil. They are also the most likely to live in neighborhoods with crumbling water and waste-management systems, or where garbage is not collected on a regular basis, increasing their exposure to zoonotic and water-borne disease.

As every student learns during the first day of any introductory public health class, public health focuses the health of a population or community. In contrast, clinical medicine involves the care of individual patients. Even when public health measures involve individuals, they are part of a group effort, such as a mass vaccination program, rather than receiving individualized medical care. In this regard, bioethics is a lot closer to public health than is at first apparent. Although clinical bioethicists are considered with the care and treatment of individual patients, other bioethicists consider more generalizable and widelyapplicable theories to address such issues such as cloning, stem cell research or organ transplantation.

So, does the urgent need to address lack of universal access to health care mean that bioethics should give up its traditional clinically-oriented role? Not necessarily, as bioethics unique approach to such problems may help offset public health's tendency to try to develop one-size-fits-all solutions for medical crises. Bioethics has played an essential role in protecting and promoting the rights of individual patients in a health care culture that traditionally has been based on the decisions of doctors. For example, the change in medical practice from the days when doctors felt comfortable in not telling patients they had cancer is enormous. Today, patients routinely have access to their own medical information and work collaboratively with their doctors in choosing or rejecting a course of treatment. It is not by pure coincidence that these changes developed along with the field of bioethics.

If bioethics prizes individuals, public health often disregards them. Rather than consider each person's psychosocial make-up, public health dictates are usually broad based. It often seems as though the recommendations of the public health community are no more individualized than the advice we all got from our mothers: wash your hands, eat your vegetables, keep out of the cookie jar, brush your teeth, take your vitamins, don't smoke and get outside and play. Research overwhelmingly shows, however, that such 
"lifestyle" factors have an enormous influence on individual health by preventing the chronic diseases to which all humans are subject.

Society can benefit by bringing these two specialties together. Bioethics has much to teach public health about respecting individual rights, even though this might not be in the best interests of a population. One of the best example of this comes from bioethics' insistence that decisions about organ transplants be made on a person-by-person basis, without considering the needs of others. A bioethicist would see nothing wrong with giving a dying child a second heart-lung transplant, even when the likelihood of a positive outcome is non-existent, whereas a person coming from a public health perspective might think about how those organs could be better used by other, healthier members of the transplant community. However, bioethics' focus on the rights and needs of individual recipients often ignores the broader question of what interventions, such as improved living conditions or timely diagnosis and treatment of chronic diseases, might reduce the need for transplants. By combining these two perspectives, bioethicists and public health professionals can give greater depth to decisions about the allocation of scarce health care resources in society, whether these resources are organs for transplant, expensive or experimental drugs, or even hospital beds. The resulting health care system would incorporate both the infrastructure to work smoothly throughout the country but still promote and protect individual humanrights.

Developing a health care system that is effective and equitable will require the contributions of many different areas of expertise. It took a Category Five Hurricane to do it, but the attention of the public is now focused on such issues as poverty and the unacceptable living conditions of their fellow citizens. We now have the rare opportunity to again consider the idea of a national health care system, one that would begin to address the inequities that are so starkly displayed on the nightly news. By combing bioethics' sensitivity to individual rights with public health's well-practiced interventions for the communal good, we can tackle the desperate and unacceptable state of the American health care system. As members of the bioethics and public health community, we can share our strength, widen our scope of interest and start closing the socioeconomic gap by providing access to a safe environment, and adequate and just health care to all Americans, not to mention all of our fellow citizens of the World.

\section{REFERENCE}

Moreno, J. D. 2005. In the wake of Katrina: has "bioethics" failed? The American Journal of Bioethics 5(5): W18-W19. 